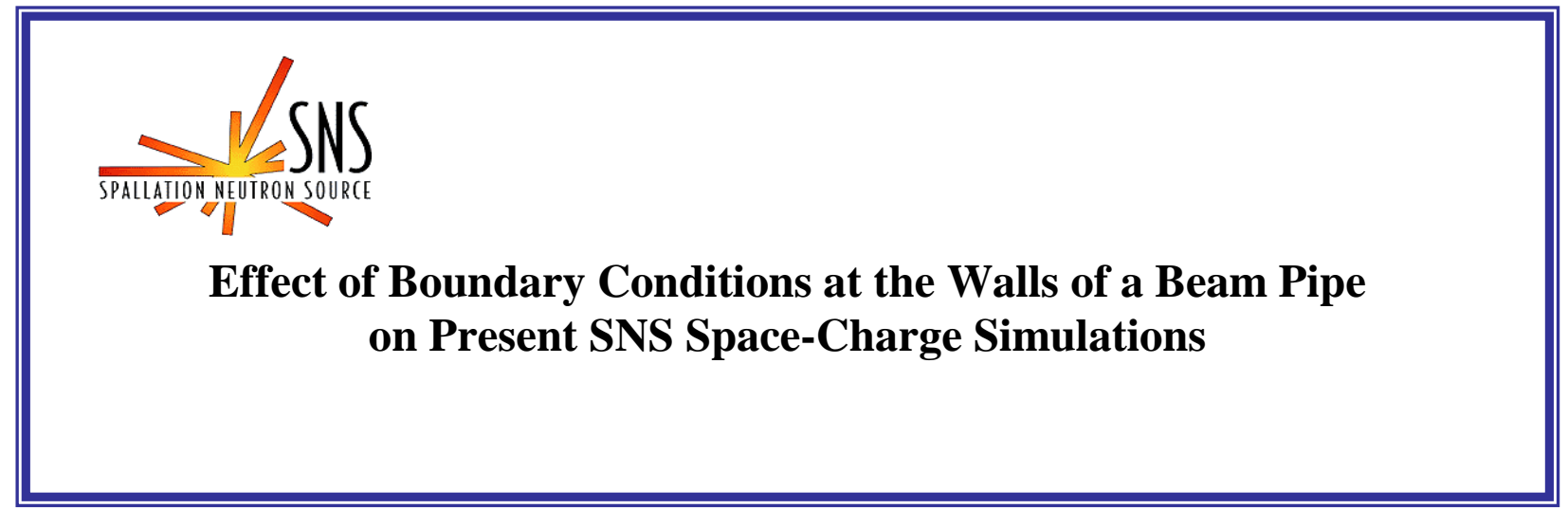

BNL/SNS TECHNICAL NOTE

NO. 078

Alexei V. Fedotov

June 1, 2000

COLLIDER-ACCELERATOR DEPARTMENT

BROOKHAVEN NATIONAL LABORATORY

UPTON, NEW YORK 11973 


\title{
Effect of boundary conditions at the walls of a beam pipe on present SNS space-charge simulations.
}

\author{
Alexei V. Fedotov \\ C-A Department, BNL, Bldg. 817, Upton, NY 11973-5000
}

(June 1, 2000)

\section{INTRODUCTION}

The present SNS space-charge simulations are performed using several computer codes: SIMPSONS [1], ORBIT [2] and UAL [3]. In SIMPSONS, the Poisson equation for the space-charge potential is solved with boundary conditions at the wall of a vacuum chamber of circular cross section. While in ORBIT and UAL, the space-charge algorithm is implemented using the Hockney convolution method [4] with open boundary condition thus missing the effect of image charges and currents. Such algorithm was chosen based on speed consideration which is a crucial question in space-charge simulations with a large number of particles.

The UAL open environment addresses a complex combination of several physical effects typical to the SNS project. Some of them are field errors, misalignments and space-charge. The greatest advantage of the UAL is that it supports the incremental development of independent components. For example, space-charge and injection painting were specifically developed for ORBIT and then implemented into UAL in a simple manner. Thus UAL has identical to ORBIT space-charge algorithm with open boundary conditions. In the same way, UAL allows straightforward inclusion of any other space-charge algorithm which will prove to be more efficient or more accurate.

The ultimate goal of our space-charge studies is to achieve very low level of beam losses. In order to be able to make predictions of the low level loss, tracking with a large number of particles becomes essential. However, before rushing in simulations with millions of particles we need to be sure that the most significant physical effects are included in our simulations. 
We thus have to identify and estimate the importance of various effects not yet included in the code. The purpose of these notes is to understand the contribution of one of such effects - the effect of walls.

To get some feeling whether wall effect can be important in our space-charge simulation we examine contributions to the incoherent and coherent tune shifts arising from the image charges and currents. We will specifically reproduce boundary conditions required for derivation of the tune shift formulas and for realistic simulations. Tune shift formulas for a general case of a bunched beam will be also presented. To our knowledge, the best collection and description of these formulas is given in [5] and [6]. Formulas for these notes are simply taken from [6] and are reproduced here for clarity and convinience of discussions. We also try to eliminate typing mistakes present in the literature.

\section{GENERAL DEFINITIONS}

In addition to direct space-charge fields, induced fields due to charges and currents in the surrounding vacuum chamber walls (image fields) shift the betatron frequencies which is often called Laslett tune shifts. Formulas below will be presented for the vertical motion, but they are also applicable to the horizontal motion.

For the vertical motion we have:

$$
\frac{d^{2} y}{d t^{2}}+Q^{2} \Omega^{2} y=F / m_{0} \gamma
$$

where $Q$ is a bare betatron tune and $\Omega$ is a revolution frequency. The function $F$ can be expanded to first order of the test particles motion $y$ and the average beam position $\bar{y}$ to give:

$$
\frac{d^{2} y}{d t^{2}}+Q^{2} \Omega^{2} y=\frac{1}{m_{0} \gamma}\left(\left[\frac{\partial F}{\partial y}\right]_{\bar{y}=0} y+\left[\frac{\partial F}{\partial \bar{y}}\right]_{y=0} \bar{y}\right) .
$$

When $\bar{y}(t)=0$, we have:

$$
\frac{d^{2} y}{d t^{2}}+\left(Q^{2} \Omega^{2}-\frac{1}{m_{0} \gamma}\left[\frac{\partial F}{\partial y}\right]_{\bar{y}=0}\right) y=0
$$


which gives the following incoherent tune shift:

$$
\Delta Q_{i n c}=-\frac{1}{2 Q m_{0} \gamma \Omega^{2}}\left[\frac{\partial F}{\partial y}\right]_{\bar{y}=0}
$$

When $d \bar{y} / d t \neq 0$, the coherent motion can be solved by choosing $y=\bar{y}$ in Eq. (2.2). We thus have:

$$
\frac{d^{2} y}{d t^{2}}+\left(Q^{2} \Omega^{2}-\frac{1}{m_{0} \gamma}\left(\left[\frac{\partial F}{\partial y}\right]_{\bar{y}=0}+\left[\frac{\partial F}{\partial \bar{y}}\right]_{y=0}\right)\right) \bar{y}=0
$$

with the coherent tune shift

$$
\Delta Q_{c o h}=-\frac{1}{2 Q m_{0} \gamma \Omega^{2}}\left(\left[\frac{\partial F}{\partial y}\right]_{\bar{y}=0}+\left[\frac{\partial F}{\partial \bar{y}}\right]_{y=0}\right)
$$

\section{BOUNDARY CONDITIONS AND FIELD COEFFICIENTS}

\section{A. Incoherent}

For the incoherent tune shift, the case is pretty simple since all the fields are static and the force $F$ is reduced to the single term $(\partial F / \partial y) y$. To obtain the self-field and image-field coefficients, we need to satisfy the following boundary conditions:

TABLE I. Field source and boundary condition

\begin{tabular}{lll}
\hline \hline Field source & boundary conditions & components in $(\partial F / \partial y)_{\bar{y}=0}$ \\
\hline self & free space & $e\left(\left[\frac{\partial E_{y}}{\partial y}\right]_{\bar{y}=0} \pm v_{s}\left[\frac{\partial B_{\perp}}{\partial y}\right]_{\bar{y}=0}\right)=$ \\
& $=\epsilon_{s c}\left[\frac{\lambda e}{\pi \epsilon_{0}}\right] \frac{1}{\gamma^{2} b^{2}}$ \\
\hline d.c. electric image & $E_{\|}=0$ on vacuum chamber & $e\left[\frac{\partial E_{y}}{\partial y}\right]_{\bar{y}=0}=\epsilon_{1}\left[\frac{\lambda e}{\pi \epsilon_{0}}\right] \frac{1}{h^{2}}$ \\
\hline d.c. magnetic image & $B_{\|}=0$ on magnetic poles & $e v_{s}\left[\frac{\partial B_{\perp}}{\partial y}\right]_{\bar{y}=0}=\epsilon_{2}\left[\frac{\lambda e}{\pi \epsilon_{0}}\right] \frac{\beta^{2}}{g^{2}}$ \\
\hline \hline
\end{tabular}

In this table $h$ is the half height of the vacuum chamber, and $g$ is the half gap of the magnet poles. For simple geometries the image coefficients are well known (see for example [5], [6]), and will be used later in these notes. 


\section{B. Coherent}

Boundary conditions now depend upon whether the oscillating field of the beam is of a low enough frequency to penetrate the vacuum chamber and to reach the magnetic poles or not. Electric fields are always considered as non-penetrating. However, for magnetic field both penetrating and non-penetrating fields are possible. For the non-penetrating fields, the magnetic image should be decomposed into its d.c. part, which is bounded on the poles, and a.c. part, which is bounded on the vacuum chamber.

TABLE II. Field source and boundary condition

\begin{tabular}{lll}
\hline \hline Field source & boundary conditions & $(\partial F / \partial y)_{\bar{y}=0}+(\partial F / \partial \bar{y})_{y=0}$ \\
\hline Penetrating mag. fields: & $E_{\|}=0$ on v. chamber & $e\left(\left[\frac{\partial E_{y}}{\partial y}\right]_{\bar{y}=0}+\left[\frac{\partial E_{y}}{\partial \bar{y}}\right]_{y=0}\right)=\xi_{1}\left[\frac{\lambda e}{\pi \epsilon_{0}}\right] \frac{1}{h^{2}}$ \\
\hline electric image & $B_{\|}=0$ on mag. poles & $e v_{s}\left(\left[\frac{\partial B_{\perp}}{\partial y}\right]_{\bar{y}=0}+\left[\frac{\partial B_{\perp}}{\partial \bar{y}}\right]_{y=0}\right)=\xi_{2}\left[\frac{\lambda e}{\pi \epsilon_{0}}\right] \frac{\beta^{2}}{g^{2}}$ \\
\hline magnetic image &
\end{tabular}

Non-penetrating mag. fields:

electric image

$$
E_{\|}=0 \text { on v. chamber } \quad e\left(\left[\frac{\partial E_{y}}{\partial y}\right]_{\bar{y}=0}+\left[\frac{\partial E_{y}}{\partial \bar{y}}\right]_{y=0}\right)=\xi_{1}\left[\frac{\lambda e}{\pi \epsilon_{0}}\right] \frac{1}{h^{2}}
$$

d.c. magnetic image $\quad B_{\|}=0$ on mag. poles $\quad e v_{s}\left[\frac{\partial B_{\perp}}{\partial y}\right]_{\bar{y}=0}=\epsilon_{2}\left[\frac{\lambda e}{\pi \epsilon_{0}}\right] \frac{\beta^{2}}{g^{2}}$

a.c. magnetic image $\quad B_{\perp}=0$ on v. chamber $e v_{s}\left[\frac{\partial B_{\perp}}{\partial \bar{y}}\right]_{y=0}=-\left(\xi_{1}-\epsilon_{1}\right)\left[\frac{\lambda e}{\pi \epsilon_{0}}\right] \frac{\beta^{2}}{h^{2}}$ 
Note that d.c. part for the non-penetrating fields is identical to the incoherent part $(\bar{y}=0)$. While for the penetrating fields we also have contribution from $\bar{y} \neq 0$.

\section{TUNE SHIFT IN BUNCHED BEAMS}

Below we remind tune shift formulas valid for a general case of a bunched beam (using $B_{f}$

for bunching factor) with neutralisation parameter $n_{e}$. We also keep the structure parameter $C_{i}$ which represents the fraction of the circumference occupied by each type of component.

$$
\Delta Q_{i n c}=-\left[\frac{N r_{0}}{\pi \beta^{2} \gamma}\right]\left(\frac{\bar{\beta}}{B_{f}}\left(\frac{1}{\gamma^{2}}-n_{e}\right) \frac{\epsilon_{s c}}{b^{2}}+\sum_{i} C_{i} \bar{\beta}_{i}\left[\frac{\left(1-n_{e}\right)}{B_{f}} \frac{\epsilon_{1, i}}{h_{i}^{2}}+\beta^{2} \frac{\epsilon_{2, i}}{g_{i}^{2}}-\beta^{2}\left(\frac{1}{B_{f}}-1\right) \frac{\epsilon_{1, i}}{h_{i}^{2}}\right]\right),
$$

where $N$ is a number of particles in a bunch, $r_{0}$ is a classical proton radius, $\beta$ is relativistic parameter, and $\bar{\beta} \approx R / Q$ is an average beta function. Here, the first term under the $\sum$ sign comes from the electric image, the second term comes from d.c. magnetic image, and the third term comes from a.c. magnetic image due to axial bunching. For the coherent tune shift, in the case of the penetrating magnetic fields, we have:

$$
\Delta Q_{c o h}^{p}=-\left[\frac{N r_{0}}{\pi \beta^{2} \gamma}\right] \sum_{i} C_{i} \bar{\beta}_{i}\left[\frac{\left(1-n_{e}\right)}{B_{f}} \frac{\xi_{1, i}}{h_{i}^{2}}+\beta^{2} \frac{\xi_{2, i}}{g_{i}^{2}}-\beta^{2}\left(\frac{1}{B_{f}}-1\right) \frac{\xi_{1, i}}{h_{i}^{2}}\right] .
$$

Once again, the first term under the $\sum$ sign comes from the electric image, the second term comes from d.c. magnetic image, and the third term comes from a.c. magnetic image due to axial bunching. For the coherent tune shift, in the case of the non-penetrating magnetic fields, we have:

$$
\Delta Q_{c o h}^{n o n-p}=-\left[\frac{N r_{0}}{\pi \beta^{2} \gamma}\right] \sum_{i} C_{i} \bar{\beta}_{i}\left[\frac{\left(1-n_{e}\right)}{B_{f}} \frac{\xi_{1, i}}{h_{i}^{2}}+\beta^{2} \frac{\epsilon_{2, i}}{g_{i}^{2}}-\beta^{2}\left(\frac{1}{B_{f}}-1\right) \frac{\xi_{1, i}}{h_{i}^{2}}-\beta^{2} \frac{\left(\xi_{1, i}-\epsilon_{1, i}\right)}{h_{i}^{2}}\right],
$$

where additional term comes from a.c. magnetic image due to the transverse motion. 


\section{APPLICATION TO SNS PARAMETERS}

Before we apply formulas (presented in the previous section) for typical SNS ring parameters, we make several assumptions. We assume that $n_{e}=0$, and also do not take into account the structure parameter $C_{i}$. Then, after rearranging, the tune shift formulas become

$$
\begin{gathered}
\Delta Q_{i n c}=-\left[\frac{N r_{0}}{\pi \beta^{2} \gamma b^{2}} \bar{\beta}\right]\left(\frac{1}{\gamma^{2}} \frac{\epsilon_{s c}}{B_{f}}+\frac{1}{\gamma^{2} B_{f}} \frac{\epsilon_{1}}{(h / b)^{2}}+\beta^{2} \frac{\epsilon_{2}}{(g / b)^{2}}+\beta^{2} \frac{\epsilon_{1}}{(h / b)^{2}}\right) \\
\Delta Q_{c o h}^{p}=-\left[\frac{N r_{0}}{\pi \beta^{2} \gamma b^{2}} \bar{\beta}\right]\left(\frac{1}{\gamma^{2} B_{f}} \frac{\xi_{1}}{(h / b)^{2}}+\beta^{2} \frac{\xi_{2}}{(g / b)^{2}}+\beta^{2} \frac{\xi_{1}}{(h / b)^{2}}\right), \\
\Delta Q_{c o h}^{n o n-p}=-\left[\frac{N r_{0}}{\pi \beta^{2} \gamma b^{2}} \bar{\beta}\right]\left(\frac{1}{\gamma^{2} B_{f}} \frac{\xi_{1}}{(h / b)^{2}}+\beta^{2} \frac{\epsilon_{2}}{(g / b)^{2}}+\beta^{2} \frac{\epsilon_{1}}{(h / b)^{2}}\right),
\end{gathered}
$$

Expression for the self-field (SF) coefficient $\epsilon_{s c}$ will depend on the beam distribution and beam shape, while image coefficients will depend on the geometry of the vacuum chamber and magnetic poles. In this example, we assume uniform density circular beam which gives $\epsilon_{s c}=1 / 2$, this is sufficient to estimate the relative effect of images compare to the self-field contribution, which is the purpose of these notes. We also assume circular geometry for the vacuum chamber which gives $\epsilon_{1}=0, \xi_{1, x, y}=1 / 2$ (with the assumption of a pencil beam; the effect of a finite beam size on the image coefficients will be discussed in the next section). (Note that SNS beam pipe cross section is actually a hexagonal which can be approximated by an ellipse with the major/minor axis ratio close to 1.4. This would give small but finite $\epsilon_{1} \sim 0.1$ [8].) For the magnetic yoke we use approximation of parallel plates which gives $\epsilon_{2, y, x}= \pm \pi^{2} / 24, \xi_{2, x}=0, \xi_{2, y}=\pi^{2} / 16$ (note that circular magnetic yoke occurs only in superconducting magnets). Once again, we present estimates only for the vertical motion. Extension to the horizontal motion is straightforward.

Taking $B_{f} \approx 1 / 2, \gamma \approx 2, \beta^{2} \approx 0.77$ and $g / b \approx 2$, we have

$$
\Delta Q_{i n c} \approx-C\left[\frac{1}{4}-\frac{0.77}{4} \frac{\pi^{2}}{24}\right],
$$

where $C$ stands for the relative coefficient in front, the first term reflects the effect of the selffields, and the second term shows the effect of d.c. magnetic image. We can see that there 
is no effect from the electric images at all because of the circular geometry of the vacuum chamber. The effect of the magnetic images is relatively small. Besides, the magnetic image effect becomes important only at low frequencies (see Appendix).

For estimates of the coherent tune shift we now have to choose an additional parameter $h / b$. The smallest ratio in the case of the correlated painting is currently $(h / b)^{2}=2$. With this value, for the penetrating magnetic fields we have:

$$
\Delta Q_{c o h}^{p} \approx-C\left[\frac{1}{8}+\frac{0.77}{4}+\frac{0.77}{4} \frac{\pi^{2}}{16}\right]
$$

where the first term reflects the effect of the electric image and a.c. magnetic image, the second term reflects a.c. magnetic image due to axial bunching, and the third term shows the effect of d.c. magnetic image. Thus, we would have important contribution from the first two terms. Similarly, for the non-penetrating fields, we have:

$$
\Delta Q_{c o h}^{n o n-p} \approx-C\left[\frac{1}{8}-\frac{0.77}{4} \frac{\pi^{2}}{24}\right]
$$

where the second term again applies only to very low frequencies, and thus important contribution will come only from the first term. With $(h / b)^{2}=4$ (which would be the largest possible ratio for the final-intensity beam in present SNS design) contribution from the first term (electric image plus a.c. magnetic image) will be $1 / 4$ of the self-field contribution, which is still significant. We thus try to summarize these effects via the following table:

\section{TABLE III. Relative effect}

\begin{tabular}{lll}
\hline \hline & Coherent tune shift & Incoherent tune shift \\
\hline Electric image & from $1 / 2$ to $1 / 4$ of the SF term & 0 (due to circular v.ch. geometry) \\
plus a.c. magnetic & & \\
\hline magnetic & d.c. magnetic: only low $f$ & small effect, plus only at low $f$ \\
penetrating & a.c. mag. from axial bunching: $1 / 2$ of SF & \\
\hline magnetic & d.c. magnetic: only low $f$ & 0 \\
non-penetrating & a.c. magnetic: 0 & \\
\hline \hline
\end{tabular}




\section{DISCUSSION OF A FINITE BEAM SIZE EFFECT}

In our estimates we used the image coefficients derived in the assumption that distances between a beam and its images are much greater than the transverse beam size. This assumption allows us to approximate the beam and its images by line charges and currents which is not quite accurate for the SNS beam parameters.

Below we underline the principle difference due to Zotter [7]. For a pencil beam at $x_{0}$ in a circular vacuum chamber of radius $h$, the electric image coefficient evaluated at point $x$ is given by

$$
\epsilon_{1}=-\frac{\phi_{0}^{2}}{2\left(1-\phi_{0} \phi\right)^{2}},
$$

where $\phi_{0} \equiv x_{0} / h$ and $\phi \equiv x / h$. For a beam at the center of vacuum chamber $\left(\phi_{0}=0\right)$, and thus we have $\epsilon_{1}=0$ as we used in our estimates. For a uniform flat beam of width $2 a$ we have [7]:

$$
\epsilon_{1}\left(\phi, \phi_{0}, \alpha\right)=-\frac{1}{2 \phi^{2}}\left[1+\frac{1}{u^{2}-v^{2}}+\frac{1}{v} \ln \frac{u-v}{u+v}\right]
$$

where $u \equiv 1-\phi \phi_{0}, v \equiv \alpha \phi$ with $\alpha \equiv a / h$. For $\phi \rightarrow 0$ this expression becomes indeterminate. Thus this limit should be obtained directly from integration over a beam of finite width. Final result is the following:

$$
\epsilon_{1}\left(0, \phi_{0}, \alpha\right)=-\frac{1}{2}\left(\phi_{0}^{2}+\frac{\alpha^{2}}{3}\right)
$$

Now, at $\phi_{0}=0, \epsilon_{1} \neq 0$, and, for example, for $\alpha^{2}=1 / 2$ we have $\epsilon_{1}=-1 / 12$.

We can see that for the final-size SNS beam at full-intensity our estimates based on pencil beams are not quite accurate. However, we should note that, due to the multi-turn injection painting, beam size increases gradually. The significant value of $\alpha$ is reached only at the end of painting just before extraction. Thus we can still neglect the finite beam size contribution in our rough estimates the purpose of which were just to get feeling of the necessity of boundary condition in present simulations. Clearly, finite beam size corrections are required for instability studies with the full-intensity beam. But we already concluded, even based on 
the assumption of a pencil beam, that exact boundary conditions in simulations are essential for instability studies.

Also note that more realistic estimates would require taking into the fact that the SNS vacuum chamber has elliptic cross section. For such vacuum chamber profile and a finite beam size it is not possible to obtain analytic expression as in the case of a pencil beam but it can be done numerically for specific parameters [7].

\section{CONCLUSIONS}

For present SNS parameters, disregarding wall effect in simulations does not have significant impact on the incoherent tune shifts and associated with them studies such as a choice of the working point, resonances or dynamic aperture.

For the coherent tune shifts and associated coherent instabilities, studies missing boundary conditions at magnetic poles would give significant effect only for low frequencies (see Appendix). However, boundary conditions at the vacuum chamber (both electric and magnetic) are important, especially for very small ratios of $h / b$.

Since at this stage we are not concerned with the instability studies based on UAL, our space-charge simulations are probably accurate enough even without wall effects. However, this question of images should be addressed when we decide to incorporate impedances for instability and realistic beam dynamics studies first in ORBIT and then in UAL.

\section{ACKNOWLEDGMENTS}

We wish to thank M. Blaskiewicz, N. Malitsky and J. Wei for helpful discussions.

\section{APPENDIX A: FREQUENCY RANGE FOR NON-PENETRATING FIELDS}

In this section we follow B. Zotter criterion for deciding whether the fields will penetrate. The fields will be non-penetrating if the skin depth $\delta<\sqrt{h w}$, where $h$ is the radius of the 
vacuum chamber and $w$ is the wall thickness. We thus have

$$
\begin{gathered}
\delta=\sqrt{\frac{c}{2 \pi \sigma k}}<\sqrt{h w}, \\
k>\frac{c}{2 \pi \sigma h w} .
\end{gathered}
$$

For the SNS, with $w=4 \mathrm{~mm}, h=10 \mathrm{~cm}$, and the conductivity of a stainless steel $\sigma=$ $1.3 * 10^{16}[1 / \mathrm{s}]$, we find that fields are non-penetrating for frequencies $f>450 \mathrm{~Hz}$. We thus refer to the frequencies below $450 \mathrm{~Hz}$ as low frequencies. Such low frequencies are outside the range of interest for the SNS.

[1] S. Machida, Nucl. Instrum. Methods, A309 (1991) p.43.

[2] J. Galambos et al., ORBIT User's Manual, SNS/ORNL/AP Technical Note 011, 1999.

[3] N. Malitsky and R. Talman, AIP 391 (1996).

[4] R.W. Hockney and J.W. Eastwood, Computer simulation using particles (Adam Hilger, New York, 1988).

[5] G. Guignard, Selection of formulae concerning proton storage rings, CERN 77-10 (June 1977).

[6] P.J. Bryant and K. Johnsen, The Principles of Circular Accelerators and Storage Rings (Cambridge University Press 1993).

[7] B. Zotter, Incoherent Q-shift of a flat, off-centered particle beam in an elliptical vacuum chamber, CERN 74-38 (July 1974).

[8] B. Zotter, Image fields of an off-center particle beam in an elliptic vacuum chamber, CERN 74-11 (February 1974). 